\title{
Catálogo de publicaciones del \\ Cechiver-UV
}

- Revista Orientando.

\section{Temas de Asia Oriental, Sociedad, Cultura y Economía}

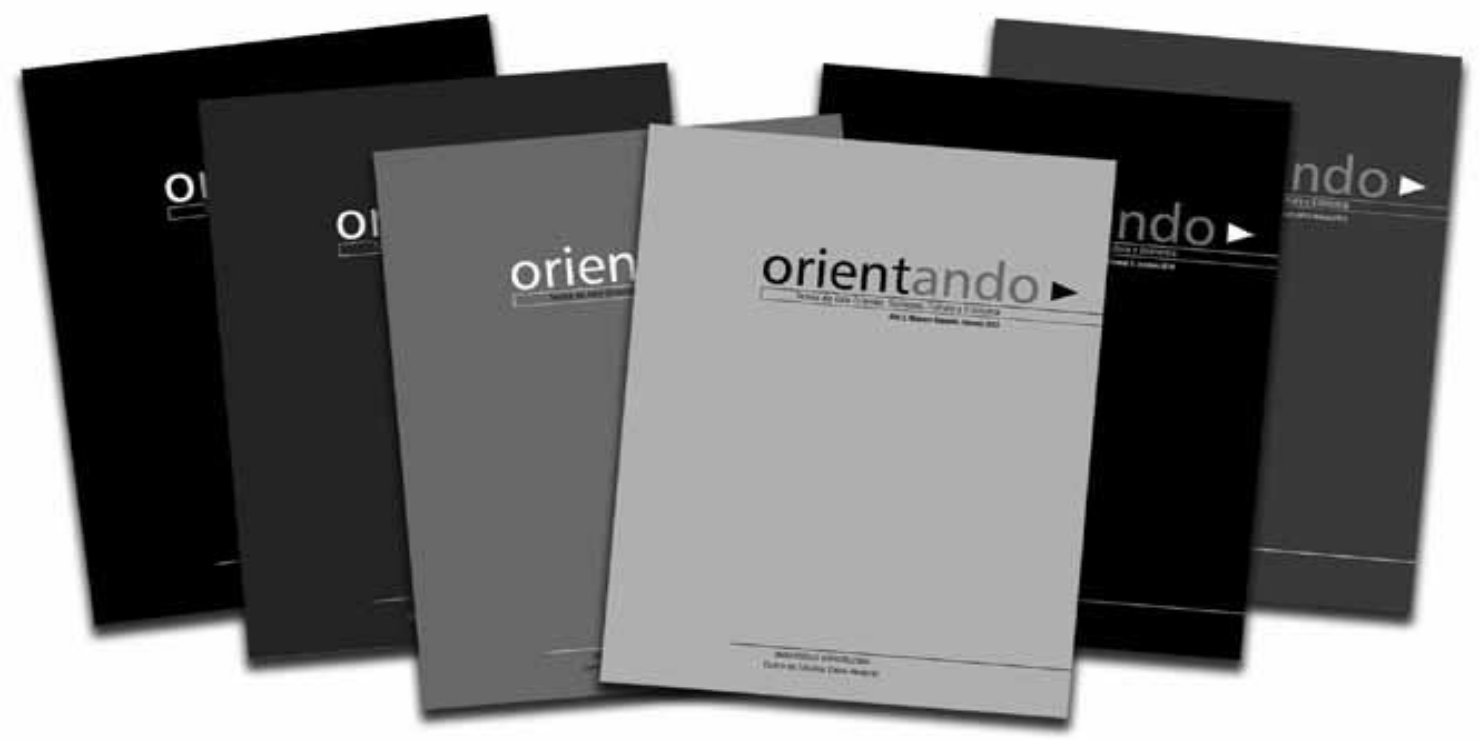

Publicación semestral del Centro de Estudios China-Veracruz de la Universidad Veracruzana.

Inicio de la publicación: octubre de 2010.

Actualmente, Orientando cuenta con 15 ediciones publicadas, hasta marzo de 2018, además del número especial dedicado a la inauguración del Centro de Estudios sobre México y América Latina "Sergio Pitol", en septiembre de 2011, en la Universidad de Ciencia y Tecnología de Chongqing, China (publicado en febrero de 2012).

Desde el primer número, la revista ha contado con la colaboración de investigadores de organismos nacionales y extranjeros, entre los que destacan: los Institutos de Relaciones Internacionales Contemporáneas de China (CICIR), la 
Universidad de Estudios Internacionales de Beijing (BISU), el Instituto de América Latina de la Academia China de Ciencias Sociales (ILAS-CASS), la Renmin Daxue (Universidad del Pueblo de China), la Universidad de Hubei (Hunan, China), el Centro de Estudios Asiáticos de la Universidad de Rice, EE. UU. y la Universidad Complutense de Madrid; además de la Universidad Nacional Autónoma de México y la Universidad Veracruzana.

La revista Orientando se publica en dos versiones: en físico (con el ISSN 20075510) y en digital (con el ISSN 2007-5723); se puede consultar en línea a través de la página: orientando.uv.mx

\section{Libro}

\section{- China: relatos del nuevo mundo}

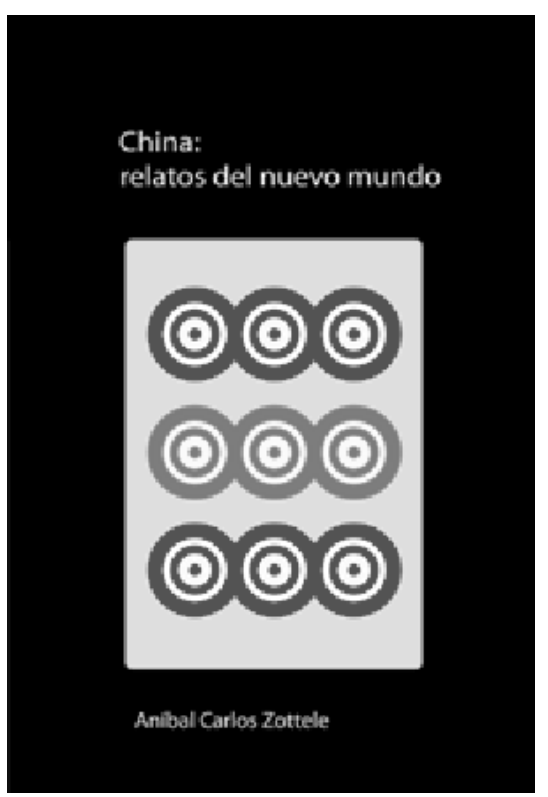

- Ficha bibliográfica:

Zottele, A. C. (2011). China:

relatos del nuevo mundo. Xalapa:

Universidad Veracruzana.
Aníbal Carlos Zottele narra en este libro una serie de relatos sobre las características del nuevo mundo. En esta obra, revisa los axiomas y las categorías que durante una larga etapa de la historia -y aún ahora- se han utilizado para "explicar" no sólo la realidad pasada y presente de China, sino de todas las naciones orientales.

Dichas premisas, que provienen del “orientalismo", una corriente de pensamiento dominante en Europa, son analizadas críticamente en el libro. Es una reflexión oportuna, en un momento en que China recupera su papel protagónico en la economía mundial y desafía la visión prejuiciosa de la comunidad internacional. 


\section{Capítulo}

- "La industrialización y urbanización de Perú, Chile, México y Argentina (1950-1970)"

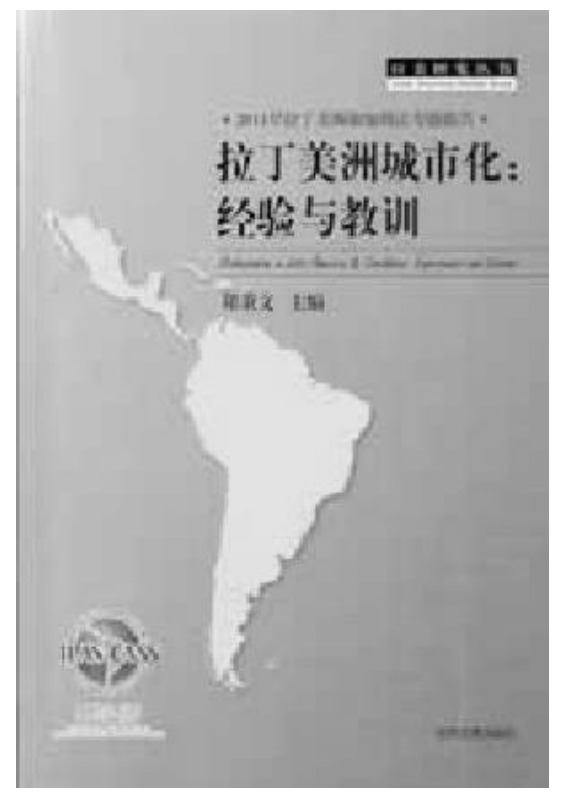

Cechiver colabora en el libro Urbanización de América Latina y el Caribe: Experiencias y lecciones, editado por el Instituto de América Latina de la Academia China de Ciencias Sociales (ILAS-CASS), con el capítulo "La industrializacón y urbanización de Perú, Chile, México y Argentina (19501970)".

El artículo, publicado en chino, es un análisis minucioso del tema, escrito por: Aníbal Carlos Zottele, Mario Alberto Santiago, Teresa Chaires y Esteban Zottele de Vega.

- Ficha bibliográfica:

Zottele, A. C., Santiago, M. A., Chaires, T., \& Esteban, Z. (2011). "La industrialización y urbanización de Perú, Chile, México y Argentina (1950-1970)". En Urbanización de América Latina y el Caribe: Experiencias y lecciones. Beijing: Instituto de América Latina de la Academia China de Ciencias Sociales (ILAS-CASS). 


\section{Libro}

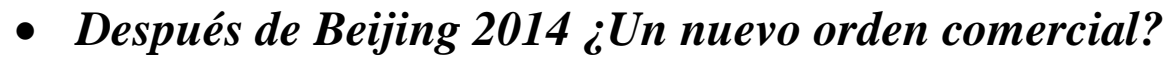

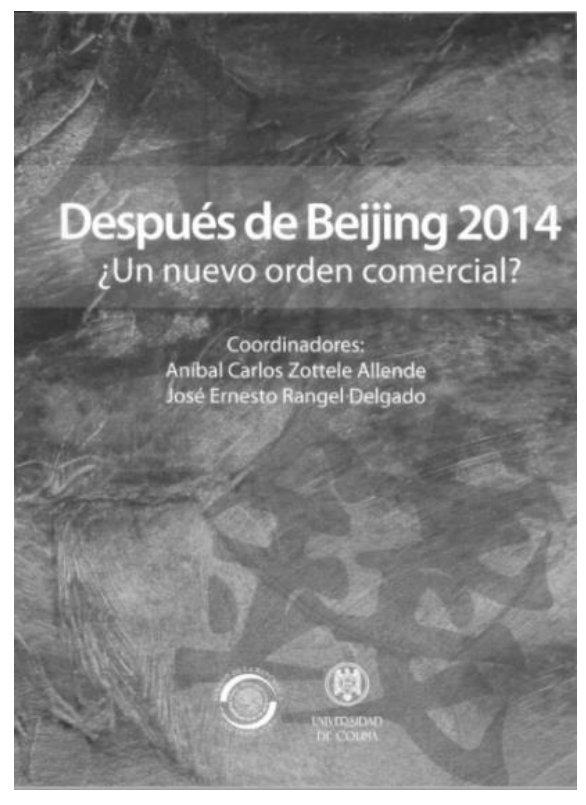

En noviembre de 2014 se realizó la 22a Cumbre de Líderes de APEC en Beijing, una de las reuniones más importantes de los últimos años. El presidente $\mathrm{Xi}$ Jinping propuso retomar una de las estratégicas más ambiciosas que se habían impulsado en dicho foro, a fin de avanzar aún más en la liberalización comercial en la región. Esta propuesta es considerada una vía alterna al bloque conformado en el Pacífico y conocido como el "Acuerdo de Asociación Transpacífico", que promueve la liberalización del comercio entre los países integrantes.

Ante este panorama, la presente obra reúne la visión y el análisis de destacados académicos y especialistas en nueve capítulos. Los autores reflexionan en torno a preguntas fundamentales y proponen una serie de medidas que México necesita desarrollar para participar dentro de las cadenas productivas de la región AsiaPacífico, en particular con China. Se estudian también las políticas públicas que ha implementado el gobierno de Xi Jinping para lograr un desarrollo en materia de ciencia y tecnología. *

*Reseña escrita por el Senador Teófilo Torres Corzo, presidente de la Comisión de Relaciones Exteriores, Asia-Pacífico.

- Ficha bibliográfica:

Zottele Allende, A. C., \& Rangel, J. E. (2016). Después de Beijing 2014 ¿Un nuevo orden comercial? México: Universidad de Colima. 


\section{Capítulo}

- "El impacto social del comercio entre China y América Latina: hacia la reformulación de los intercambios”

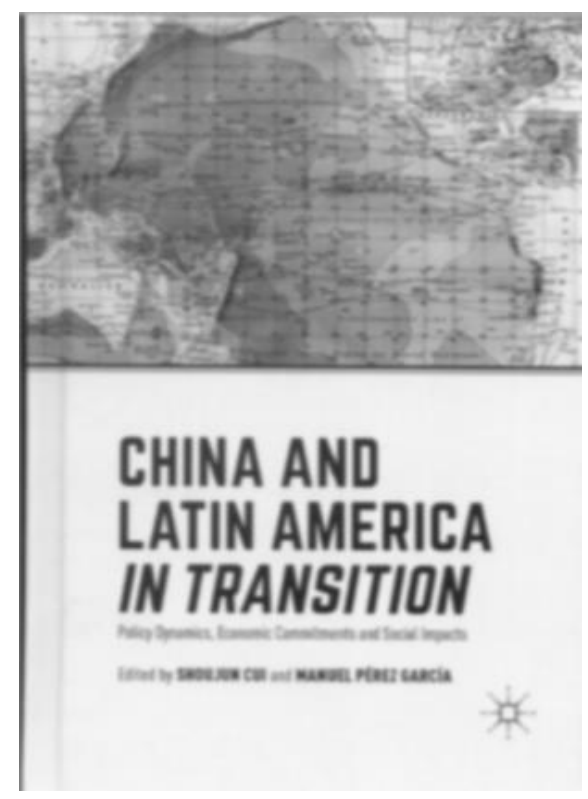

Este volumen explora las políticas, los compromisos económicos y los impactos sociales de la rápida evolución de las relaciones sino latinoamericanas; así como el vínculo de China con América Latina y el Caribe. En este título, Aníbal Carlos Zottele publica el artículo "El impacto social del comercio entre China y América Latina: hacia la reformulación de los intercambios".

Mientras China se ha comprometido a fortalecer sus lazos económicos y políticos con América Latina y el Caribe, AL está repitiendo el esfuerzo, desviando su enfoque hacia el otro lado del océano. El aspecto transicional de estos lazos es fenomenal y se manifiesta no sólo en el momento acelerado del comercio, la inversión y el préstamo, sino también en el mecanismo del Foro China-CELAC.

En tanto que AL se redefine como una prioridad emergente para el liderazgo en Beijing, ¿cuáles son las respuestas de esta región y de Estados Unidos? En esta obra, expertos de cuatro continentes proporcionan respuestas locales a una pregunta global.

- Ficha bibliográfica:

Zottele, A. C. (2016). "The Social Impact of the Commercial Trade Between China and Latin America: Toward the Reformulation of Exchanges". En China and Latin America in Transtion. EE. UU: Palgrave Macmillan. 


\section{Libro}

- Las Pymes mexicanas y chinas

ante el crecimiento acelerado de las relaciones económicas entre ambas naciones

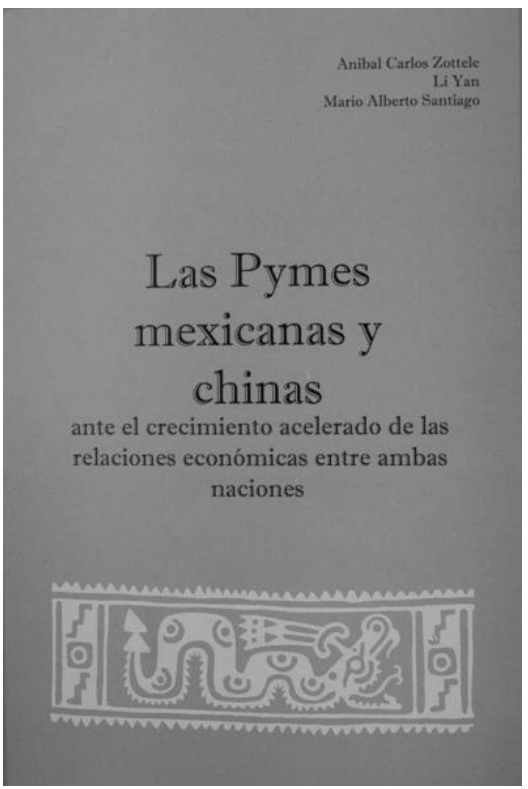

Publicado por Cechiver, a través de la Dirección General de Relaciones Internacionales (DGRI) y de la Cámara de Comercio de México en China (Mexcham).

En este título, los autores Aníbal Carlos Zottele, Li Yan y Mario Alberto Santiago, analizan un tema muy importante para el panorama económico y político actual: la relación entre China y México en el contexto mundial del libre comercio.

Es el resultado de una intensa investigación sobre el desarrollo de las pequeñas y medianas empresas, desde una perspectiva histórica. Constituye una referencia necesaria para analizar los retos que enfrentan las Pymes hoy en día; un punto de partida para que la comunidad académica genere propuestas que impulsen el fortalecimiento y la innovación de las mismas.

- Ficha bibliográfica:

Zottele Allende, A. C., Li, Y., \& Mario Alberto, S. (2017). Las Pymes mexicanas $y$ chinas ante el crecimiento acelerado de las relaciones económicas entre ambas naciones. Xalapa: Universidad Veracruzana. 


\section{Artículo}

- "Diversificación del comercio exterior veracruzano: oportunidades en el sudeste asiático"

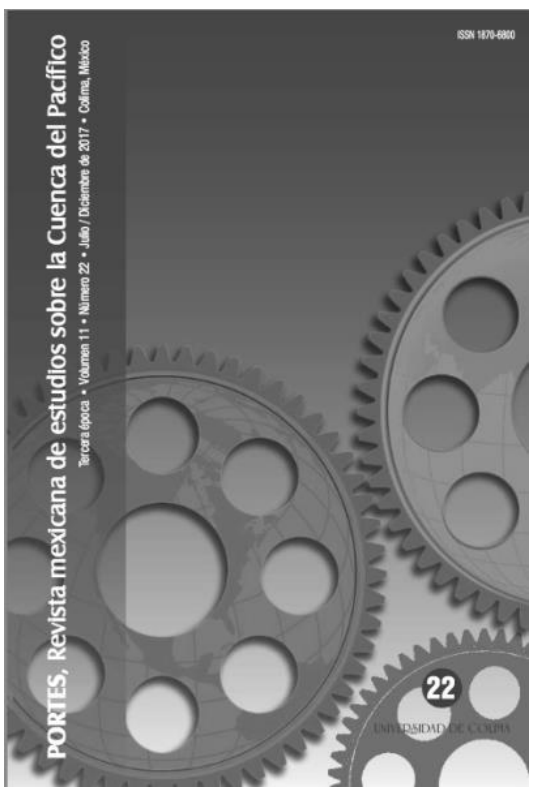

En el Número 22 de Portes, Revista mexicana de estudios sobre la Cuenca del Pacífico (juliodiciembre de 2017), Aníbal Carlos Zottele, Abiagil Vázquez Morales y Érika Irais Benítez Arcos, publican el artículo "Diversificación del comercio exterior veracruzano: Oportunidades en el sudeste asiático".

Los autores reflexionan sobre cómo los empresarios veracruzanos se encuentran en condiciones de diversificar el destino de su producción e ingresar a la región del sudeste asiático, que no sólo representa una oportunidad extraordinaria, sino que se proyecta como uno de los mayores demandantes de bienes y servicios en el futuro próximo.

- Ficha bibliográfica:

Zottele Allende, A. C., Vázquez Morales, A., \& Érika Irais, B. (Julio-diciembre de 2017). "Diversificación del comercio exterior veracruzano: oportunidades en el sudeste asiático". En Portes. Revista mexicana de estudios sobre la Cuenca del Pacífico, 11(22). 


\section{Libro}

- Universidad Veracruzana: una década de experiencia en China

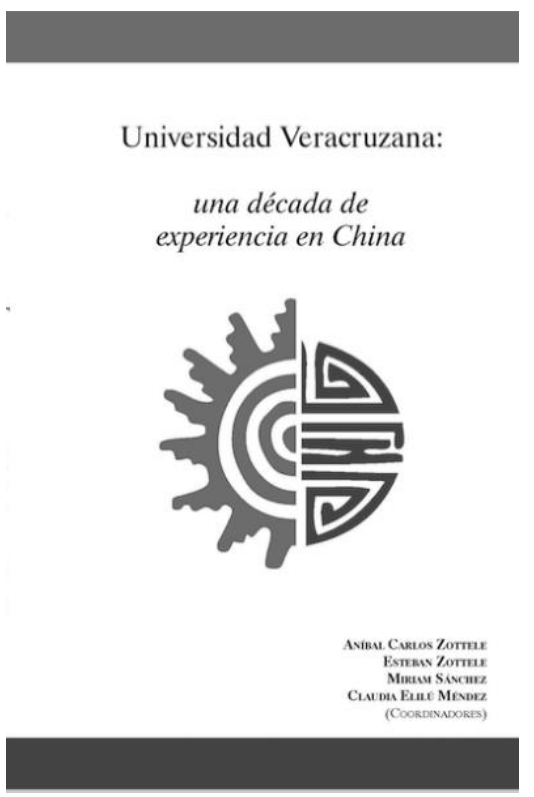

Desde la creación del Cechiver en el año 2008, decenas de estudiantes, académicos y empresarios del estado de Veracruz y del resto del país han sido parte de los programas desarrollados por la Universidad Veracruzana en el marco de la relación China-América Latina. Actualmente, los lazos entre la UV y entidades educativas, así como organismos públicos y privados del "país del centro", son privilegiados.

Este libro es el anecdotario de una década de intercambio entre dos grandes pueblos, que a través de la educación y la cultura han hallado las claves para consolidar un enlace fecundo y nutrir el paso de un nuevo ritmo histórico.

- Ficha bibliográfica:

Zottele, A. C., Zottele, E., Sánchez, M., \& Claudia, M. (2018). Universidad Veracruzana: una década de experiencia en China. Xalapa: Universidad Veracruzana. 